\title{
SOME EXAMPLES OF MODULES OVER NOETHERIAN RINGS
}

\author{
by I. M. MUSSON
}

(Received 23 April, 1980)

1. Introduction. The purpose of this note is to prove the following result.

THEOREM 1. Let $n$ be an integer greater than zero. There exists a prime Noetherian ring $R$ of Krull dimension $n+1$ and a finitely generated essential extension $W$ of a simple $R$-module $V$ such that

(i) $W$ has Krull dimension $n$, and

(ii) $W / V$ is $n$-critical and cannot be embedded in any of its proper submodules.

We refer the reader to [6] for the definition and properties of Krull dimension.

Theorem 1 answers questions of Jategaonkar and Goldie. Let $R$ be a two-sided Noetherian ring. In [7] Jategaonkar asks whether every finitely generated essential extension of a simple $R$-module is artinian, and Goldie [4] asks whether a critical $R$-module is necessarily compressible.

The ring $R$ is the enveloping algebra of a certain finite dimensional metabelian Lie algebra.

Finitely generated, non-artinian essential extensions of simple $R$-modules were studied in [8] for the case where $R$ is a polycyclic group algebra. An example of a 1-critical module which is not compressible was found independently by Goodearl [5]. This example closely resembles our module $W / V$ for the case $n=1$.

We note that the bounds on Krull dimension are best possible for a prime Noetherian ring $R$ of Krull dimension $n+1$. For, by [8, Proposition 5.5], a finitely generated essential extension of a simple $R$-module can have Krull dimension at most $n$, while [6, Proposition 6.8 states that an $n+1$-critical $R$-module is isomorphic to a right ideal of $R$ and so cannot have the property expressed in (ii).

A simplified version of this example (the case $n=1$ ) is to appear in [2, Chapter 7]. I am very grateful for the hospitality of the University of Alberta where this work was completed.

2. The example. Let $k$ be a field of characteristic zero and $\mathscr{L}$ a vector space over $k$ with basis $y, x_{0}, x_{1}, \ldots, x_{n-1}$.

We make $\mathscr{L}$ into a Lie algebra by defining

$$
\begin{aligned}
& {\left[x_{i} x_{j}\right]=0 \quad\left[x_{0} y\right]=x_{0} } \\
& {\left[x_{i} y\right]=x_{i}+x_{i-1} \text { for } i=1, \ldots, n-1 . }
\end{aligned}
$$

Let $R$ be the universal enveloping algebra of $\mathscr{L}$. Then $R$ is a prime Noetherian ring of Krull dimension $n+1$, by [3, $\S \S 2.3$ and 3.5].

Let $I=\sum_{i=0}^{n-1}(y-1)\left(x_{i}-1\right) R$ and $W=R / I$. For each non-negative integer $m$ we set

$$
v_{m}=(y-1) y^{m}+I \in W .
$$

Glasgow Math. J. 23 (1982) 9-13. 
Then $v_{m}=v_{0} y^{m}$ and we have

$$
v_{0} x_{i}=v_{0}
$$

since $(y-1) x_{i}-(y-1) \in I$. Set $V=v_{0} R$, then $v_{m} \in V$ for all $m$, and $V$ is spanned as a vector space by $\left\{v_{m}: m \geq 0\right\}$.

Lemma 1. The $R$-module $V$ is simple.

Proof. We show by induction that

$$
v_{m}\left(1-x_{0}\right)^{m}=m ! v_{0}
$$

Suppose that $v_{m}\left(1-x_{0}\right)^{m}=m ! v_{0}$. Then by (2) and (3),

$$
\begin{aligned}
v_{m+1}\left(1-x_{0}\right)^{m+1} & =v_{m} y\left(1-x_{0}\right)^{m+1} \\
& =v_{m}\left(y-y x_{0}\right)\left(1-x_{0}\right)^{m} \\
& =v_{m}\left(y-x_{0} y+x_{0}\right)\left(1-x_{0}\right)^{m} \\
& =v_{m}\left(1-x_{0}\right) y\left(1-x_{0}\right)^{m}+v_{m} x_{0}\left(1-x_{0}\right)^{m} \\
& =v_{m}\left(1-x_{0}\right)\left(y-x_{0} y+x_{0}\right)\left(1-x_{0}\right)^{m-1}+m ! v_{0} \\
& =v_{m}\left(1-x_{0}\right)^{2} y\left(1-x_{0}\right)^{m-1}+2 m ! v_{0}=\ldots \\
& =v_{m}\left(1-x_{0}\right)^{m+1} y+(m+1) m ! v_{0} \\
& =m ! v_{0}\left(1-x_{0}\right) y+(m+1) ! v_{0} \\
& =(m+.1) ! v_{0} .
\end{aligned}
$$

Hence (3) holds for all $m$. It follows that if $v \in V, v \neq 0$ then $v_{0} \in v R$, so $V$ is simple. Another easy consequence of (3) is that the $v_{i}$ form a vector space basis for $V$. In order to state the next lemma we introduce some notation. If $e=\left(e_{0}, e_{1}, \ldots, e_{n-1}\right)$ is an $n$-tuple of non-negative integers we denote by $x^{e}$ the monomial

$$
x_{0}^{e_{0}} x_{1}^{e_{1}} \ldots x_{n-1}^{e_{n-1}}
$$

Also, let $J=(y-1) R$. Then $J \supseteq I$ and $J / I=V$.

LEMma 2. (i) Let $e=\left(e_{0}, e_{1}, \ldots, e_{r}, 0, \ldots, 0\right)$. Then the following identity holds in $R$.

$$
x^{e} y=\left(y+\sum_{i=0}^{r} e_{i}+\sum_{i=1}^{r} e_{i} x_{i-1} x_{i}^{-1}\right) x^{e}
$$

(ii) Modulo $J$ we have

$$
x^{e}\left(y-1-\sum_{i=0}^{r} e_{i}\right) \equiv \sum_{i=1}^{r} e_{i} x_{i-1} x_{i}^{-1} x^{e} .
$$

Here the notation $x_{i}^{-1}$ is purely symbolic. Thus if $e_{i}=0$ this term does not appear, while if $e_{i}>0$ then $x_{i}^{-1} x^{e}=x^{f}$ where $f_{j}=e_{j}$ if $j \neq i$ and $f_{i}=e_{i}-1$. 
Proof. (ii) follows immediately from (i) since $(y-1) \in J$.

(i) The defining relations (1) tell us how $y$ may be moved to the left past any $x_{i}$ and this result records how y may be moved past any monomial. We use induction on $r$ and for a fixed $r$, induction on the exponent $e_{r}$.

Thus let $f_{i}=e_{i}$ if $i \neq r$ and $f_{r}=e_{r}+1$. Then

$$
\begin{aligned}
x^{f} y & =x^{e} x_{r} y=x^{e}\left(y x_{r}+x_{r}+x_{r-1}\right) \\
& =\left(y+\sum_{i=0}^{r} e_{i}+\sum_{i=1}^{r} e_{i} x_{i-1} x_{i}^{-1}\right) x^{e} x_{r}+x^{e} x_{r}+x^{e} x_{r-1} \\
& =\left(y+\sum_{i=0}^{r} e_{i}+1+\sum_{i=1}^{r} e_{i} x_{i-1} x_{i}^{-1}+x_{r-1} x_{r}^{-1}\right) x^{f} \\
& =\left(y+\sum_{i=0}^{r} f_{i}+\sum_{i=1}^{r} f_{i} x_{i-1} x_{i}^{-1}\right) x^{f}
\end{aligned}
$$

as required.

Notice that the module $R / J=W / V$ has a basis consisting of elements $x^{e}+J$ and it is immediate from Lemma 2 that when $\left(x^{e}+J\right) y$ is written as a linear combination of elements $x^{f}+J$, the exponent sum on each $x^{f}$ is the same as on $x^{e}$.

To gain further information from Lemma 2 it is convenient to introduce an ordering on monomials $x^{e}$.

Thus we write $x^{f}<x^{e}$ if for some $i \geq 0, e_{i}-f_{i}>0$ and $e_{i+1}-f_{i+1}=\ldots=e_{n-1}-f_{n-1}=0$.

Note that any collection $\left\{x^{e}\right\}$ of monomials has a unique element which is minimal under this ordering. Also if $\alpha=\sum \lambda_{f} x^{f}$ is a non-zero linear combination of monomials then since $\operatorname{Supp} \alpha$ is finite there is a unique monomial in Supp $\alpha$ which is maximal under this ordering. We denote this monomial by $\max \alpha$.

Finally if $\alpha$ is an arbitrary element of $R$ and $\alpha \notin J$ then $\alpha$ is uniquely representable in the form $\alpha \equiv \sum \lambda_{f} x^{f} \bmod J$ and we set $\max \alpha=\max \left(\sum \lambda_{f} x^{f}\right)$.

Lemma 3. Suppose that $\alpha=\sum \lambda_{f} x^{f}$ and $\max \alpha=x^{e}$ where $e=\left(e_{0}, e_{1}, \ldots, e_{n-1}\right)$ satisfies $e_{i}>0$ for some $i \geq 1$. If

$$
\beta=\alpha\left(y-1-\sum_{i=0}^{n-1} e_{i}\right)
$$

then $\beta \notin J$ and $\max \beta<\max \alpha$.

Proof. Let

$$
\alpha=\sum_{x^{j}<x^{e}} \lambda_{f} x^{f}+\lambda_{e} x^{e}
$$

and let $i$ be the least integer greater than 0 with $e_{i}>0$.

By Lemma $2 \max x^{e}\left(y-1-\sum_{i=0}^{n-1} e_{i}\right)=x^{\mathrm{g}}$ where $g_{i-1}=e_{i-1}+1, g_{i}=e_{i}-1$ and $g_{j}=e_{j}$ for $j \neq i, i-1$. 
Since the monomials $x^{f}$ are linearly independent modulo $J$, in order to show that $\beta \notin J$ it suffices to show that $x^{8}$ cannot occur in Supp $x^{f}\left(y-1-\sum_{i=0}^{n-1} e_{i}\right)$ for any $x^{f}<x^{e}$ and $x^{f} \in \operatorname{Supp} \alpha$.

Notice that this can only possibly occur if $\sum_{i=0}^{n-1} e_{i}=\sum_{i=0}^{n-1} f_{i}$ and in this case we would have $x^{\mathrm{g}}=x^{h}$ where for some $k, h_{k-1}=f_{k-1}+1, h_{k}=f_{k}-1, h_{l}=f_{l}, l \neq k, k-1$.

Suppose first that $k>i$. Then $f_{k}-1=e_{k}$ so $f_{k}>e_{k}$ and $e_{k+1}-f_{k+1}=\ldots e_{n-1}-f_{n-1}=0$. This contradicts the maximality of $x^{e}$ in Supp $\alpha$.

Suppose that $k<i$. Then $f_{k-1}+1=e_{k-1}$, and since $k-1<i$ we have $e_{k-1}=0$. Therefore $f_{k-1}=-1$, another contradiction.

Hence $k=i$, but in this case $f_{i-1}+1=e_{i-1}+1, f_{i}-1=e_{i}-1$ and $f_{j}=e_{j}$ if $j \neq i, i-1$ and so $x^{f}=x^{e}$.

We have shown that the term $x^{8}$ occurs with non-zero coefficient in $\beta$.

To see that $\max \beta<\max \alpha$ note that if $x^{f}<x^{e}$ then any element $x^{8} \in$ Supp $x^{f}\left(y-1-\sum_{i=0}^{n-1} e_{i}\right)$ satisfies $x^{g} \leq x^{f}$ by Lemma 2 .

Lemma 4. The module $W$ is an essential extension of $V$.

Proof. Let $T$ be a right ideal of $R$ which strictly contains $I$. We must show that $J \subseteq T$. If $T$ contains a non-zero element of $J$ we are finished since $J / I$ is simple by Lemma 1 .

Hence we may assume that $T$ contains an element $\alpha=\sum \lambda_{f} x^{f}+r$ where $r \in J$ and $\sum \lambda_{f} x^{f} \neq 0$. Among such elements $\alpha$ choose $\alpha \in T$ with $\max \alpha$ minimal, say

$$
\alpha=\sum_{x^{f}<x^{e}} \lambda_{f} x^{f}+\lambda_{e} x^{e}+r .
$$

If $e=\left(e_{0}, e_{1}, \ldots, e_{n-1}\right)$ and $e_{i}>0$ for some $i \geq 1$ then Lemma 3 immediately gives a contradiction to the minimality of $\max \alpha$.

Therefore $T$ contains an element of the form $\lambda_{0} x_{0}^{s}+\ldots+\lambda_{t} x_{0}^{s+t}+r$ with $r \in J, \lambda_{t} \neq 0$, $\lambda_{0} \neq 0 t \geq 0$. If $t$ is chosen minimal then Lemma 2 gives $t=0$.

Hence $T / I$ contains an element $x_{0}^{s}+r+I$ where $s \geq 1$ and $r \in J$. Therefore

$$
\left(x_{0}^{s}+r+I\right)(y-1-s)=(y-1) x_{0}^{s}+r(y-1-s)+I=v_{0}+r(y-1-s)+I \in(J / I) \cap(T / I) .
$$

By writing $r$ as a linear combination of the elements $v_{i}$, it is easy to see that this is a non-zero element of $V$. Hence $V \cap(T / I) \neq 0$.

Proof of Theorem 1. It remains to show that $W / V$ is $n$-critical and cannot be embedded in any of its proper submodules.

Let $k X$ denote the subalgebra of $R$ which is generated by $x_{0}, x_{1}, \ldots, x_{n-1}$. Then the $R$-module $\bar{W}=W / V$ is free as a $k X$-module. We use induction on $n$ to show that a non-zero $R$-module $\bar{W}$ which is free as a $k X$-module has Krull dimension at least $n$.

Let $K=x_{0} R$, a 2 -sided ideal of $R$, and consider the chain $\bar{W}>\bar{W} K>\bar{W} K^{2}>\ldots$. For $n=1$ this chain shows that $\bar{W}$ has Krull dimension at least 1 . Assume $n>1$. Then 
$\bar{W} K^{m} / \bar{W} K^{m+1}$ is a non-zero free $R / K$-module for each $m$. The ring $R / K$ has exactly the same defining relations as $R$ except that the parameter $n$ has dropped to $n-1$. (This is because $x_{0}=0$ gives $\left[x_{1} y\right]=x_{1}$ and $\left[x_{i} y\right]=x_{i}+x_{i-1}$ if $i>1$.)

Therefore by induction $\bar{W} K^{m} / \bar{W} K^{m+1}$ has Krull dimension at least $n-1$ and so $\bar{W}$ has Krull dimension at least $n$.

If we regard $W / V$ simply as a $k X$-module then $W / V$ is free of rank one. Hence as $k X$ is a commutative Noetherian domain of Krull dimension $n$, it follows that $W / V$ is $n$-critical as a $k X$-module and hence also as an $R$-module.

Finally, to see that $W / V=R / J$ cannot be embedded in any proper submodule, notice that by Lemma 2 , the only element of $R / J$ which is annihilated by $y-1$ is $1+J$. This completes the proof of Theorem 1 .

The case $n=1$ of Theorem 1 may be of special interest. In this case $\mathscr{L}$ has the form

$$
\mathscr{L}=k x_{0} \oplus k y \text { where }\left[x_{0} y\right]=x_{0}
$$

and if $k$ is algebraically closed then $\mathscr{L}$ is an epimorphic image of any finite dimensional soluble Lie algebra which is not nilpotent [1, p. 71]. Also in this case it is easily seen that the module $W=R /(y-1)\left(x_{0}-1\right) R$ obtained in Theorem 1 is uniserial, that is every non-zero submodule of $W$ has a unique maximal submodule. Hence we may state

THEOREM 2. Let $k$ be an algebraically closed field of characteristic zero and $\mathscr{L}$ a finite dimensional soluble Lie algebra over $k$ which is not nilpotent. Let $R$ be the enveloping algebra of $\mathscr{L}$. Then there is a finitely generated (uniserial) essential extension $W$ of a simple $R$-module $V$ such that

(i) $W$ is not artinian, and

(ii) $W / V$ is 1-critical and cannot be embedded in any proper submodule.

\section{REFERENCES}

1. W. Bohro, P. Gabriel and R. Rentschler, Primideale in Einhüllenden auflösbarer LieAlgebren Lecture Notes in Mathematics No. 357, (Springer-Verlag, 1973).

2. A. W. Chatters and C. R. Hajarnavis, Rings with chain conditions (Pitman, 1980).

3. J. Dixmier, Enveloping algebras (North Holland, 1977). 1972).

4. A. W. Goldie, Properties of the idealiser, in Ring theory, Ed. R. Gordon (Academic Press,

5. K. R. Goodearl, Incompressible critical modules, Comm. Algebra, 8 (1980), 1845-1852.

6. R. Gordon and J. C. Robson, Krull dimension, Memoirs Amer. Math. Soc. 133 (1973).

7. A. V. Jategaonkar, Jacobson's conjecture and modules over fully bounded Noetherian rings, J. Algebra 30 (1974), 103-121.

8. I. M. Musson, Injective modules for group rings of polycyclic groups II, Quart. J. Math. Oxford (2), 31 (1980), 449-466.

Department OF Mathematics

UNIVERSITY OF AlBERTA

EDMONTON

Canada T6G $2 \mathrm{G} 1$.

\author{
Present address: \\ Department of Mathematics \\ UNIVERSITY OF WISCONSIN-MADISON \\ Madison, Wisconsin 53706
}

